\title{
Non-intercepting electron beam transverse diagnostics with optical diffraction radiation at the DESY FLASH facility ${ }^{2}$
}

\author{
E. Chiadroni ${ }^{\text {a,* }}$, M. Castellano ${ }^{\text {a }}$, A. Cianchi ${ }^{\text {b }}$, K. Honkavaara ${ }^{\text {c }}$, G. Kube ${ }^{\text {, }}$, \\ V. Merlo ${ }^{\mathrm{e}}$, F. Stella ${ }^{\mathrm{e}}$

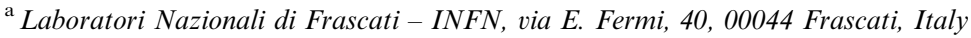 \\ b INFN di Roma "Tor Vergata", via della Ricerca Scientifica, 1, 00133 Roma, Italy \\ ${ }^{\mathrm{c}}$ Universität Hamburg, Inst. f. Experimentalphysik, Luruper Chaussee 149, 22761 Hamburg, Germany \\ ${ }^{\mathrm{d}}$ Deutsches Elektronen-Synchrotron, Notkestraße 85, 22607 Hamburg, Germany \\ e Università di Roma "Tor Vergata", via della Ricerca Scientifica, 1, 00133 Roma, Italy
}

Received 3 December 2007; received in revised form 5 February 2008

Available online 29 February 2008

\begin{abstract}
Linear colliders (LCs) and short wavelength free electron lasers (FELs) require ultra-high brilliant electron beams of so much power density that no intercepting device can sustain it. The development of suitable beam diagnostics, non-invasive and non-intercepting, is necessary to measure the properties of such beams.

Optical diffraction radiation (ODR) is considered as one of the most promising candidates, as testified by the interest of many laboratories all around the world.

An experiment based on the detection of ODR has been set-up at DESY FLASH Facility and it is under operation to measure the electron beam transverse parameters. The radiation is emitted by a high energetic electron beam passing through a sub-millimeter slit, opened on a screen made of aluminum deposited on a silicon substrate. Radiation is then detected by a low noise, high sensitivity CCD camera.
\end{abstract}

We report here the preliminary results for a $680 \mathrm{MeV}$ electron beam energy going through a $0.5 \mathrm{~mm}$ slit. (C) 2008 Elsevier B.V. All rights reserved.

PACS: $01.30 . \mathrm{Cc}$

Keywords: Optical diffraction radiation; DESY; Beam diagnostics; FEL

\section{Introduction}

The development of high energy LCs [1] and short wavelength FELs [2-4] requires high quality electron beams, which means small transverse emittance $(<1 \mathrm{~mm} \mathrm{mrad})$ and high peak current $(\approx k A)$. Due to the large power den-

\footnotetext{
Work supported by the European Community Infra-structure Activity under the FP6 "Structuring the European Research Area" program (CARE, contract number RII3-CT-2003-506395).

* Corresponding author. Tel.: +39 0694032465; fax: +390694032565.

E-mail address: enrica.chiadroni@lnf.infn.it (E. Chiadroni).
}

sity of this kind of beams, a non-intercepting diagnostics needs to be developed and applied.

In 1996 one of the authors suggested a new method for the non-intercepting measurement of beam size, both transverse [7] and longitudinal [8]. The idea is based on the observation of diffraction radiation (DR) emitted by a charged particle beam going through a slit in a metallic foil due to the interaction of the charge electromagnetic (EM) field with the screen surface.

Since the beam goes through the slit, DR is a non-intercepting diagnostics and, therefore, excellent to be used parasitically without disturbing the electron beam. The great 
interest in this type of radiation [5] comes also from both the possibility of generation of intense radiation beams in millimeter and sub-millimeter wavelength region and beam diagnostics based on both incoherent and coherent diffraction radiation [10], depending on the longitudinal beam size with respect to the emitted wavelength.

The aim of our experiment is measuring the transverse beam size and divergence, in order to calculate the transverse emittance, by studying the angular distribution of optical diffraction radiation (ODR).

The DR angular distribution is produced by the interference of radiation from both edges of the slit. The visibility of the interference fringes is correlated to the beam size. The effect is also affected, in a slightly different way, by the angular divergence of the beam: the ODR angular distribution becomes wider and the intensity of the minimum higher, when the beam divergence increases as pointed out in Section 2. A dedicate analysis of the radiation angular distribution allows then to separate the two effects. If the beam is in a waist on the DR screen, the transverse emittance can be derived with a single non-intercepting measurement.

Our experiment is carried out at FLASH, Free electron LASer in Hamburg, at DESY (Hamburg, Germany). For this kind of experiment, FLASH is an excellent facility because due to the superconducting accelerator technology, it can drive long train pulses, up to 800 bunches per macropulse, which means high charge operation, good long term stability, small beam transverse emittance, $<2 \mathrm{~mm} \mathrm{mrad}$ and high energy, up to $1 \mathrm{GeV}$.

In this paper, we report the status of ODR experiment and we show the results from the preliminary studies on the data collected so far.

\section{DR theory}

Diffraction radiation is produced when a charged particle goes through a slit or passes by the edge of a metallic screen, due to the interaction between the EM field of the traveling charge and the target surface [9]. The intensity of the radiation increases linearly with the charge and is proportional to $\mathrm{e}^{-\frac{2 \pi a}{\gamma \hbar}}$, where $a$ is the slit aperture, $\gamma$ the Lorentz factor and $\lambda$ the emitted wavelength. A natural unit of measure of this phenomenon is given by the radial extension of the EM field, $\frac{\gamma \lambda}{2 \pi}$. In case of $a \gg \frac{\gamma \lambda}{2 \pi}$, the aperture is much greater than the extent of the particle EM field, which does not interact with the target at all and, therefore, no radiation is produced. In the opposite case, if $a \ll \frac{\gamma \lambda}{2 \pi}$, transition radiation is substantially emitted. In the following we treat the case of $a \cong \frac{\gamma \lambda}{2 \pi}$ resulting in the emission of DR.

Typically, the transverse beam size is of the order of $\mathrm{mm}$ or sub-mm. This means that in case of high energy, $\gamma \approx 10^{3}$, also optical wavelengths are emitted, allowing an easier detection of radiation, thanks to the wide instrumentation available and the reconstruction of beam transverse parameters like position, transverse size, angular divergence.

\subsection{Theoretical background}

The radiation emission mechanism can be described by the Weizsäcker-Williams method or virtual photon method [9]. The electromagnetic field of an ultra-relativistic $(\gamma \gg 1)$ particle acquires the properties of electromagnetic waves, allowing to be approximated as the superposition of plane waves (virtual photons), and can be written as

$E_{x, y}^{0}(\omega, x, y, z)=\frac{\mathrm{e} \omega}{\pi v^{2} \gamma} \frac{x, y}{\sqrt{x^{2}+y^{2}}} K_{1}\left(\frac{\omega \sqrt{x^{2}+y^{2}}}{v \gamma}\right) \mathrm{e}^{\mathrm{i} \frac{\omega}{w} z}$,

$K_{1}$ the modified Bessel function of second order. The electric field transverse components are then proportional to $\xi K_{1}(a \xi)$. A strong field is then detected and DR emitted if $a \xi=\frac{a \omega}{\gamma v}=\frac{2 \pi a}{\gamma \lambda} \leqslant 1$, where $\beta \approx 1$ has been considered. Radiation is, therefore, confined within a disk of radius $\gamma \lambda$ and, contrary to the plane electromagnetic wave, the particle field depends on the distance from it.

The diffraction radiation field can, therefore, be considered as the scattering of the virtual photon field on the metallic target. The radiated energy is determined by solving the Kirchoff integral [11] which, in the far field approximation, can be considered as the Fourier transform of the field of virtual photons on the target surface.

The ODR far field angular distribution in the Fraunhofer approximation is given by

$E_{x, y}\left(k_{x}, k_{y}\right)=-\frac{1}{(2 \pi)^{2}} \int_{S} E_{x, y}^{0}(x, y) \mathrm{e}^{-\mathrm{i}\left(k_{x} x+k_{y} y\right)} \mathrm{d} S$,

where $k_{x}$ and $k_{y}$ are the transverse components of the radiation momentum $k=\omega / c$ and $E_{x, y}^{0}$ the fields on the metallic surface given by Eq.1. For an electron moving through the center of a slit in a perfectly conducting, infinite screen, the expression for both polarizations, parallel and orthogonal to the slit, have the form

$E_{x}\left(k_{x}, k_{y}\right)=\frac{\mathrm{ie} k_{x}}{4 \pi^{2} c f}\left\{\frac{\mathrm{e}^{-\frac{a}{2}\left(f-\mathrm{i} k_{y}\right)}}{f-\mathrm{i} k_{y}}+\frac{\mathrm{e}^{-\frac{a}{2}\left(f+\mathrm{i} k_{y}\right)}}{f+\mathrm{i} k_{y}}\right\}$,
$E_{y}\left(k_{x}, k_{y}\right)=\frac{\mathrm{e}}{4 \pi^{2} c}\left\{\frac{\mathrm{e}^{-\frac{a}{2}\left(f-\mathrm{i} k_{y}\right)}}{f-\mathrm{i} k_{y}}-\frac{\mathrm{e}^{-\frac{a}{2}\left(f+\mathrm{i} k_{y}\right)}}{f+\mathrm{i} k_{y}}\right\}$,

where $f=\sqrt{k_{x}^{2}+\frac{\omega^{2}}{(v v)^{2}}}$.

The DR spectral angular distribution is then given by

$\frac{\mathrm{d}^{2} U}{\mathrm{~d} \omega \mathrm{d} \Omega}=\frac{\mathrm{d}^{2} U_{\|}}{\mathrm{d} \omega \mathrm{d} \Omega}+\frac{\mathrm{d}^{2} U_{\perp}}{\mathrm{d} \omega \mathrm{d} \Omega}=\frac{1}{4 \pi \varepsilon_{0}} \frac{\omega^{2}}{4 \pi^{2} c}\left[\left|E_{x}\right|^{2}+\left|E_{y}\right|^{2}\right]$.

The angular distribution of the DR is mainly affected by beam parameters in the plane orthogonal to the slit aperture: when the transverse beam size is increased, the intensity of both the peaks and the minima increases, resulting in the reduction of the interference fringes visibility, as stated by a simulation plotted in Fig. 1(a). Keeping in mind that the radiation angular distribution is centered around the specular direction of the particle trajectory, to evaluate the effect of the angular divergence for a real beam the DR angular distribution is convoluted with a 1D Gaussian dis- 


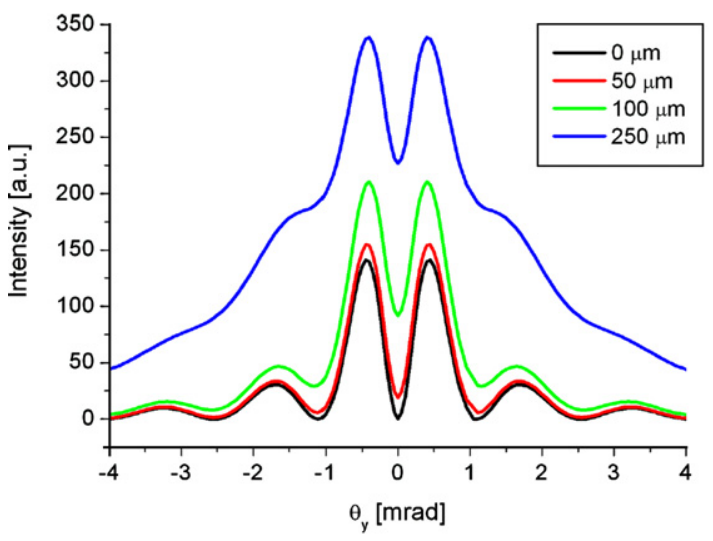

(a)

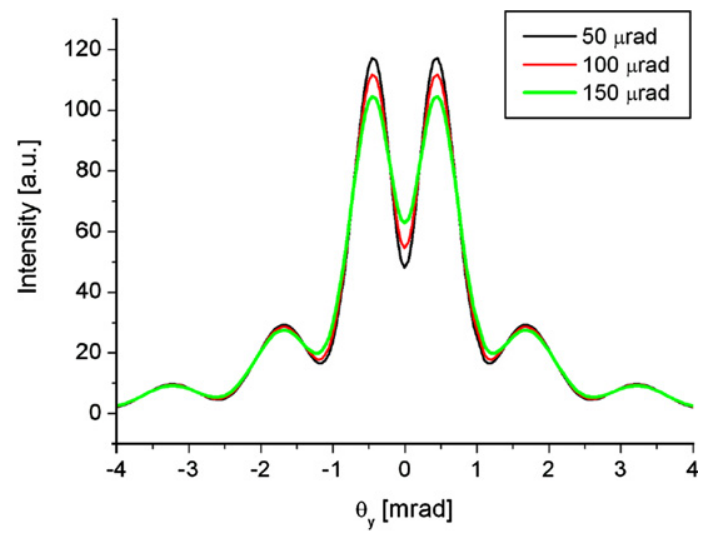

(b)

Fig. 1. ODR angular distribution for a beam of $680 \mathrm{MeV}$ energy passing through a $0.5 \mathrm{~mm}$ slit for different beam sizes (a) and beam angular divergences (b).

tribution over the variable $y$, i.e. $f\left(y, \sigma_{y}^{\prime}\right)=\frac{1}{\sqrt{2 \pi} \sigma_{y}^{\prime}} \mathrm{e}^{-\frac{y^{2}}{2 \sigma_{y}^{\prime 2}}}$. The result of the numerical computation, shown in Fig. 1(b), appears as the smoothing out of the maximum and minimum values as the beam angular divergence increases.

In our case, the slit is a horizontal rectangular aperture, allowing to measure the beam size and the beam angular divergence in the vertical plane, $\sigma_{y}$ and $\sigma_{y}^{\prime}$, respectively.

\section{Experimental apparatus}

Most of the time FLASH is operated as a user facility providing FEL radiation in the wavelength range from vacuum ultraviolet to soft X-rays for FEL experiments. Since FEL radiation is produced when a high quality electron beam passes through an undulator, the operation of FLASH is optimized to provide the required beam properties at the entrance of the undulator. Our experimental setup is mounted on the by-pass beam line (Fig. 2) in order to minimize the contribution of synchrotron light from dipole magnets, which bend the electron beam to the dump when the transport through the undulator is not required. Since the electron beam is optimized for FEL operation, tuning is typically necessary to provide proper electron beam properties on the location of our experiment.

During measurements reported in this paper, FLASH was operated with $680 \mathrm{MeV}$ electron beam energy, close

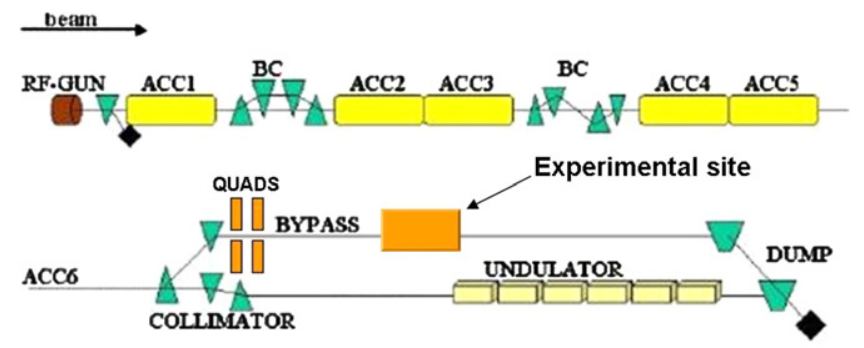

Fig. 2. FLASH layout and experimental site.

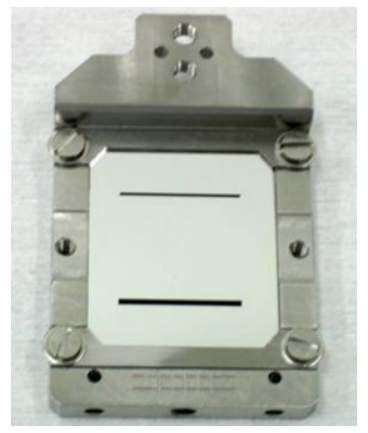

Fig. 3. DR target.

to the maximum beam energy available at that time, with only five superconducting accelerator modules.

The experimental set-up has an aluminated silicon screen (DR screen) mounted in the vacuum pipe at $45^{\circ}$ angle with respect to the beam direction. A dedicated optical system, described in Section 3.2, has been studied to guide and focus the radiation onto the camera.

\subsection{Diffraction radiator}

The target is of fundamental importance for the success of the experiment, since damaged edges and/or an uneven surface may change the interference effects, resulting in a smeared out angular distribution. Therefore, special care has been taken when manufacturing the target as well as during installation and measurements to avoid damages.

The DR screen (Fig. 3) is constructed by lithographic technique starting from a silicon nitride wafer and opening two slits, $0.5 \mathrm{~mm}$ and $1 \mathrm{~mm}$ aperture, by means of chemical etching. The slits are separated by a $2 \mathrm{~cm}$ of free screen for standard OTR beam imaging. An aluminum layer is deposited on the target by sputtering and the reflectivity is enhanced by a factor of 2 . The main advantage of the silicon nitride with respect to $\mathrm{SiO}_{2}$ [12] is a much less etching rate which preserves the silicon substrate from damages and makes the surface much more uniform. 


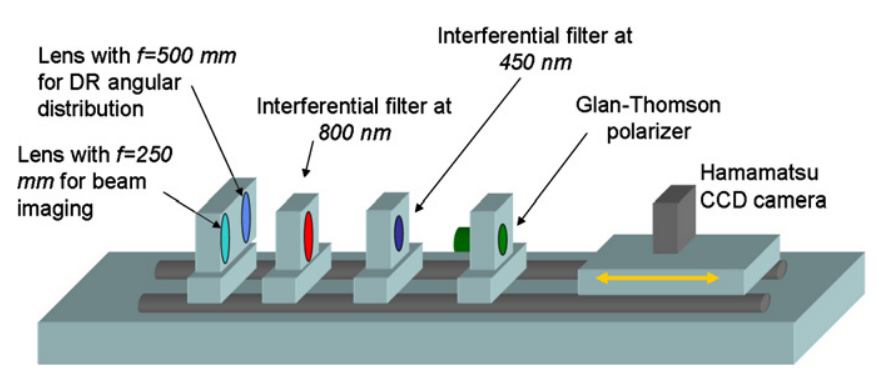

Fig. 4. Sketch of the optical system.

A motorized actuator allows a high precise positioning of the screen, down to $1 \mu \mathrm{m}$ and a very minimal movement, for accurate centering of the slit with respect to the beam.

\subsection{Light optics and acquisition system}

The optical system layout is shown in Fig. 4.

Radiation from the target is reflected by a mirror and sent through an optical system to the camera. Since the reflection power of the mirror surface is different for the horizontal and vertical polarizations and the component parallel to the incident plane (the horizontal one) is reduced, the effect is a non-perfect annular OTR angular distribution.

Two lenses can be selected: an achromat doublet to image the beam and a biconvex lens with broadband anti-reflection coating to produce the DR angular distribution. They have different focal length in order to focus on the same plane. Two interferential filters, at $800 \mathrm{~nm}$ and $450 \mathrm{~nm}$ and a Glan-Thomson polarizer may be inserted on the optical axis. The position of these elements after the lenses has been dictated by practical considerations due to the limited space. The effect of the polarizer is to lengthen the optical path, thus increasing the focal length in a range which is within the movement of the camera.

Due to the very low radiation intensity, a cooled, high sensitivity, 16-bit CCD camera ${ }^{1}$ is used. The camera main features are the very high quantum efficiency in the whole visible spectrum, in particular at $800 \mathrm{~nm}$, the negligible thermal noise and the long exposure time, up to $2 \mathrm{~h}$. Let us note that since the camera is rotated by $90^{\circ}$, vertical on the screen corresponds to horizontal and vice versa.

The optical system is remotely controlled by an electronic box, using can-bus modules, partially integrated in the linac control system and placed in the tunnel. The image acquisition and all related controls are driven via Firewire by a dedicated industrial PC located nearby and connected to the standard Ethernet network.

\section{Preliminary results}

The main limitation during the measurements was given by the synchrotron radiation background.

\footnotetext{
${ }^{1}$ Hamamatsu C4742-98-LGLAG2.
}

The beam optics was adjusted during the measurements in order to minimize the beam size in the vertical plane. To do so, since the beam parameters in the by-pass line are not well optimized, the strength of the quadrupole magnets upstream of our experiment were much stronger than the nominal values. Consequently, the contribution of the synchrotron light came not only from the dipole, but also from those quadrupoles and due to multiple reflections in the vacuum pipe, the background is the image of a source apparently near (few meters) to the target itself, clarifying its peculiar shape and preventing from the knowledge of a theoretical behavior which would be easily subtracted.

The preliminary results obtained with a $680 \mathrm{MeV}$ electron beam energy going through a $0.5 \mathrm{~mm}$ slit are reported here. FLASH was operated with up to 25 electron bunches (0.7 nC per bunch) per macropulse with $1 \mathrm{MHz}$ bunch spacing. The macropulse repetition rate was $5 \mathrm{~Hz}$.

\subsection{The beam}

A big effort was done to minimize the beam dimensions on our screen in order to let it go through the $0.5 \mathrm{~mm}$ slit. Finally, we succeeded in squeezing it only vertically, horizontally being extremely wide. The image of the beam and its intensity projections, both the horizontal and vertical one, are shown in Fig. 5(a) and Fig. 5(b), respectively. Assuming the beam is Gaussian distributed, the retrieved vertical rms size is about $80 \mu \mathrm{m}$, while the horizontal one is about ten times bigger.

\subsection{Imaging of the $O D R$ source}

A vertical scan of the slit edge was done in order to align the beam in the slit center with high accuracy and so to have an incontrovertible evidence of ODR. This kind of measurement we call near-field imaging in the sense that we focus onto the object where the ODR source is produced [6]. It allowed us to look at the dependence of the radiation intensity on the impact parameter. Since the intensity of the radiation is proportional to $\mathrm{e}^{-\frac{2 \pi a}{\nu \hbar}}$, the signal intensity is weaker as the impact parameter increases: the way this intensity goes to zero gives an indication on the kind of radiation detected.

Fig. 6(a) shows the beam through the center of the $0.5 \mathrm{~mm}$ slit in the image plane, corresponding to an impact parameter of $250 \mu \mathrm{m}$, while Fig. 6(b) corresponds to an impact parameter of $0 \mu \mathrm{m}$ (the center of the beam hits the edge of the slit). On the right edge of the slit in Fig. 6(a), a strong background radiation is visible. For this reason, the results we report here are done by scanning the slit from a position where the beam is in the center of the slit to the position where the center of the beam hits the left edge of the slit (Fig. 6(b)).

The projection of the DR near-field image is shown in Fig. 7(a) for several impact parameters compared also to the projection of the OTR image (black straight curve). The intensity of the peaks is plotted as a function of the 


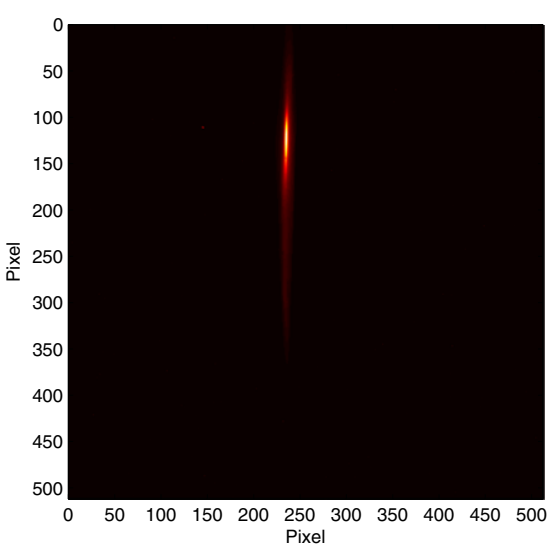

(a) OTR image of the beam

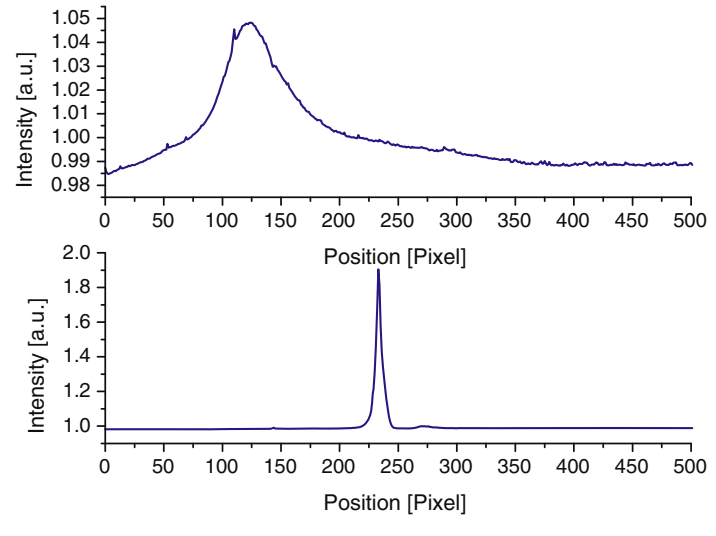

(b) Horizontal and vertical projection

Fig. 5. Characterization of the beam: single bunch, $0.7 \mathrm{nC}$ per bunch. The pixel size is $24.0 \mu \mathrm{m}(\mathrm{H}) \times 24.0 \mu \mathrm{m}(\mathrm{V})$.

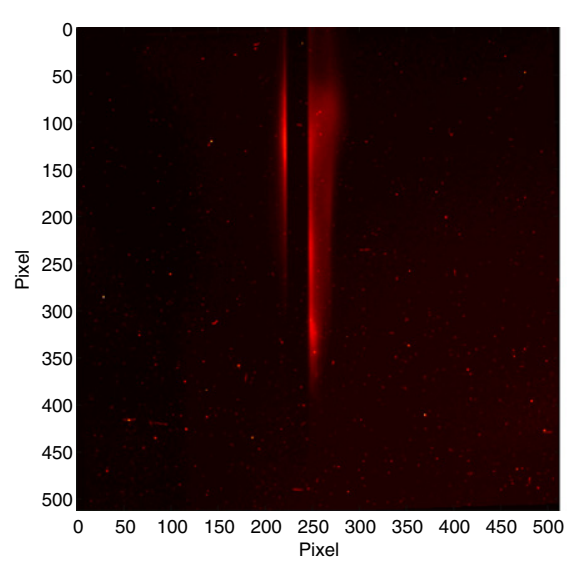

(a) Beam in the center of the slit

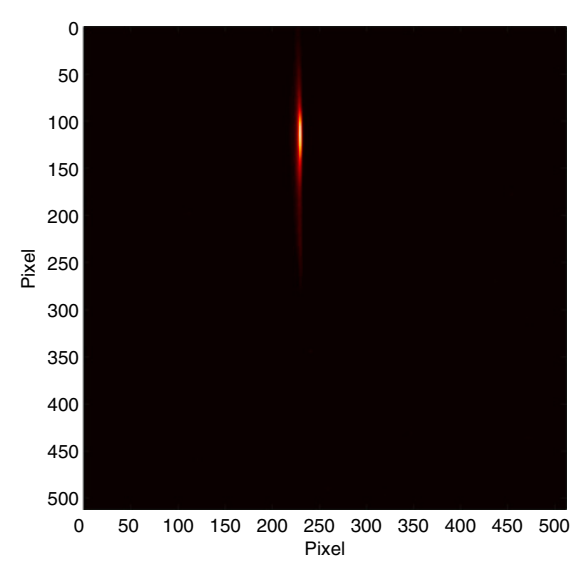

(b) Beam on one edge of the slit

Fig. 6. Near-field ODR images: the optical system is focused on the ODR source itself. The pixel size is $24.0 \mu \mathrm{m}(\mathrm{H}) \times 24.0 \mu \mathrm{m}(\mathrm{V})$.

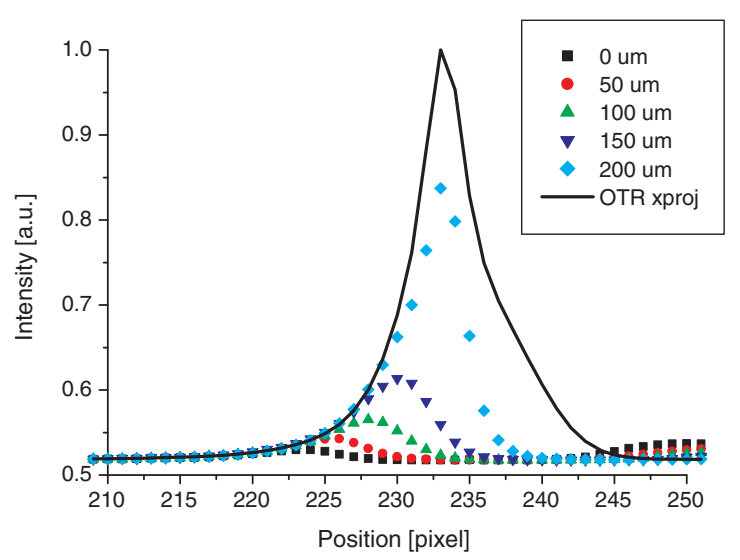

(a) Projection of the near-field image for different impact parameters

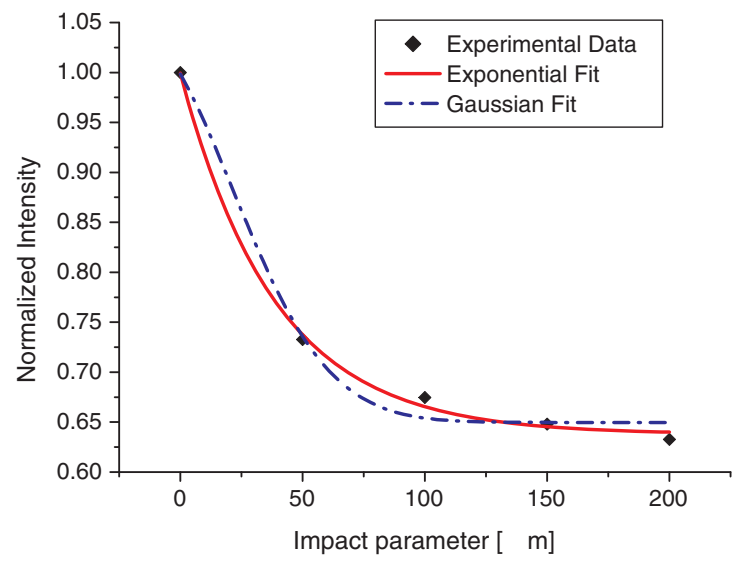

(b) Peak intensity as a function of the impact parameter

Fig. 7. Analysis of the ODR near-field images.

impact parameter in Fig. 7(b). The data are fitted with both an exponential and a Gaussian curve, in order to understand and try to distinguish, the contribution due to diffraction radiation from the one due to the tail of the beam.
Since we do not have any independent way, but OTR imaging, to verify the beam dimensions, we cannot be sure that the beam tail does not hit at all the slit edge. A slightly better agreement of the data with the exponential fit supports 


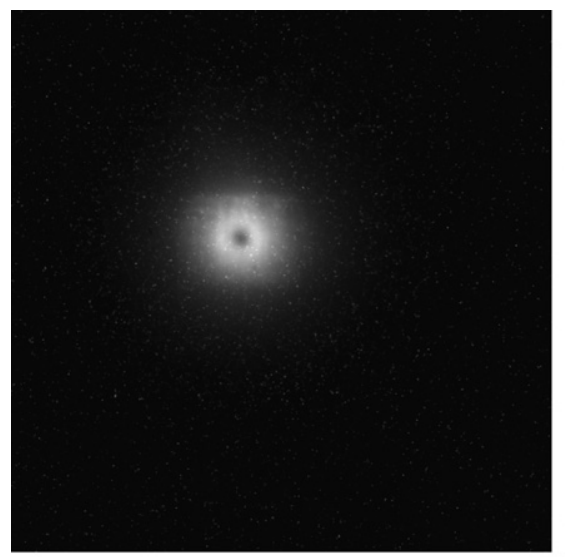

(a) OTR angular distribution

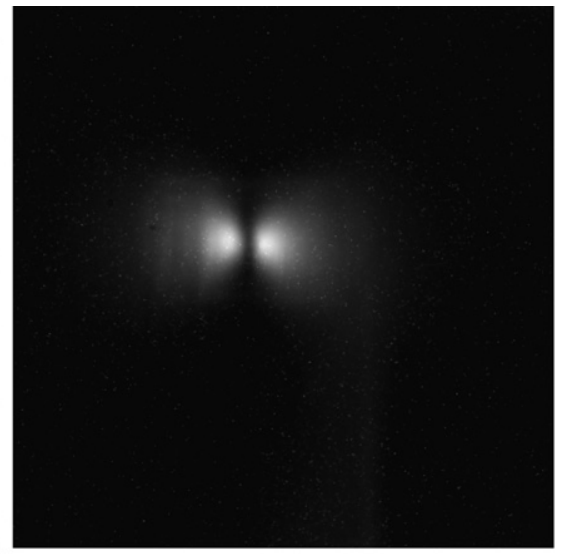

(b) OTR angular distribution with the polarizer

Fig. 8. OTR angular distribution: 10 bunches per macropulse, $0.7 \mathrm{nC}$ per bunch, $2 \mathrm{~s}$ exposure time, $5 \mathrm{~Hz}$ repetition rate.

an exponential-like decay of ODR peaks intensity as expected from theory, though a contribution of the tails cannot be excluded.

\subsection{The OTR angular distribution: A measurement of the beam energy}

Since optical transition radiation (OTR) is theoretically and experimentally well understood, we first verified our experimental setup by acquiring the OTR angular distribution, without and with the polarizer (Fig. 8) and measuring from that the beam energy. The uncertainty on the beam energy with respect to the one measured by the shift crew is about $10 \%$, compatible with the uncertainty on both the focal length and the position of the focal plane introduced by the polarizer. The disagreement on the minimum depth between the measured and the simulated curves (Fig. 9) is attributed to both, a residual background, which is also evident from the shoulders of the distribution and the angular divergence of the beam. The simulation has been done assuming a Gaussian distributed beam with a vertical angular divergence of $125 \mu \mathrm{rad}$.

The beam energy measured from the OTR angular distribution is about $619 \mathrm{MeV}$, contrary to $680 \mathrm{MeV}$ measured with standard techniques.

\subsection{Far field DR: The ODR signature}

To take a snapshot of a clear signature of ODR angular distribution, several images of both signal and background have been acquired, with the camera in the focal plane, scanning vertically the slit aperture.

Fig. 10 shows the vertically polarized angular distribution for three values of displacement of the beam with respect to one edge of the slit. The corresponding measured projection of the angular distribution is shown in Fig. 11(a). The black squares curve corresponds to the

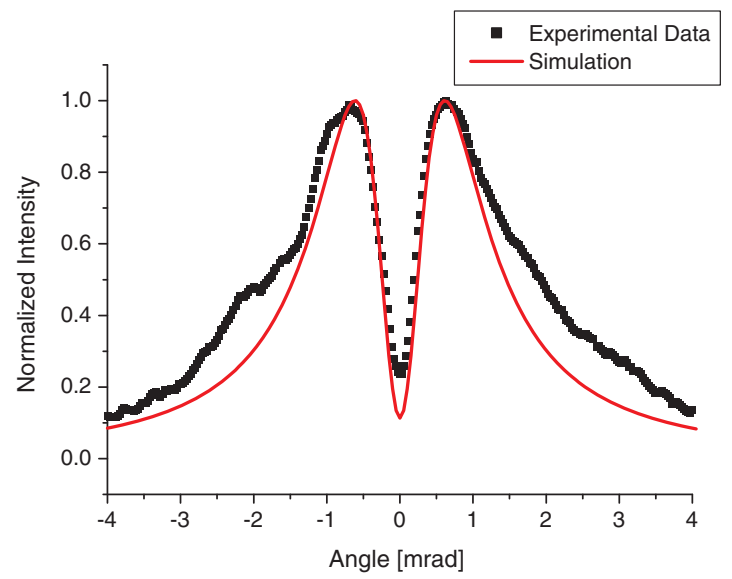

Fig. 9. Projection of the OTR angular distribution shown in Fig. 8(b) compared with a simulation done assuming a Gaussian distributed beam with vertical rms size of $80 \mu \mathrm{m}$ and vertical angular divergence of $125 \mu \mathrm{rad}$.

beam in the center (ODR). As we move far from the center towards the edge, both the minimum and the maximum intensities increase and the visibility of the interference fringes becomes less pronounced, as also shown by the simulation (Fig. 11(b)). The noisy and asymmetric curves are due to a residual background contribution.

The intensity of the minimum has been plotted as a function of the position of the beam within the whole slit (a $0 \mu \mathrm{m}$ offset corresponds now to the beam in the center of the slit). As expected from theory, Fig. 12 shows that the minimum intensity is detected when the beam is in the center of the slit. In optimum conditions, in particular zero angular divergence and with a better background subtraction, this result might be used as independent measure to determine the beam size [13].

From the previous scan we determined the center of the slit and we performed a dedicated measurement of ODR signal and background to optimize the subtraction proce- 


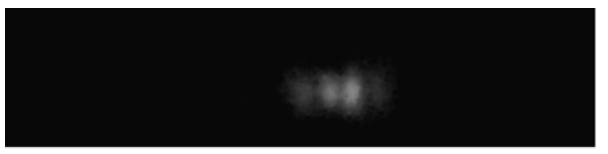

(a) $250 \mu \mathrm{m}$

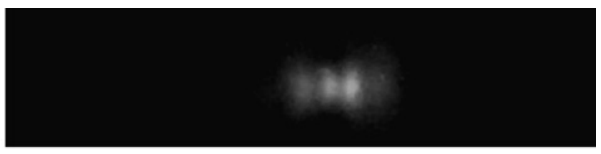

(b) $150 \mu \mathrm{m}$

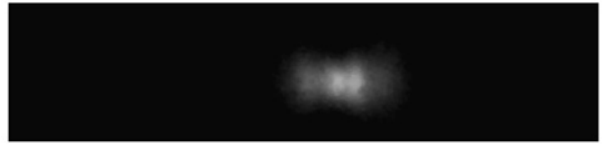

(c) $50 \mu \mathrm{m}$

Fig. 10. Vertically polarized angular distribution for different position of the beam within the slit: $250 \mu \mathrm{m}$ corresponds to the beam in the center of the slit.

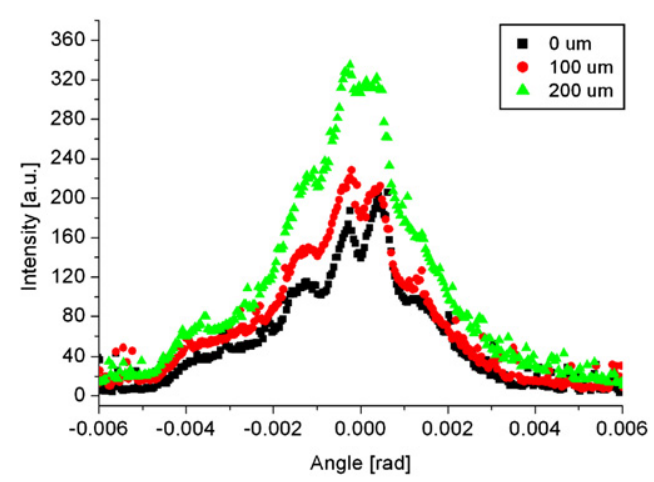

(a) Experimental data

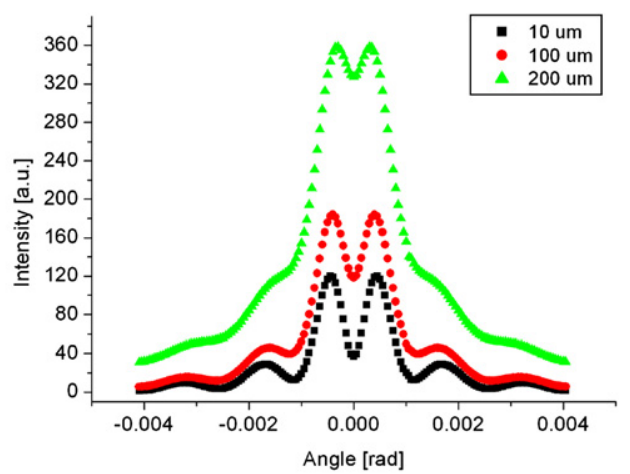

(b) Simulation

Fig. 11. Angular distributions for different positions of the beam with respect to one edge of the slit. Both the polarizer and the $800 \mathrm{~nm}$ filter are inserted.

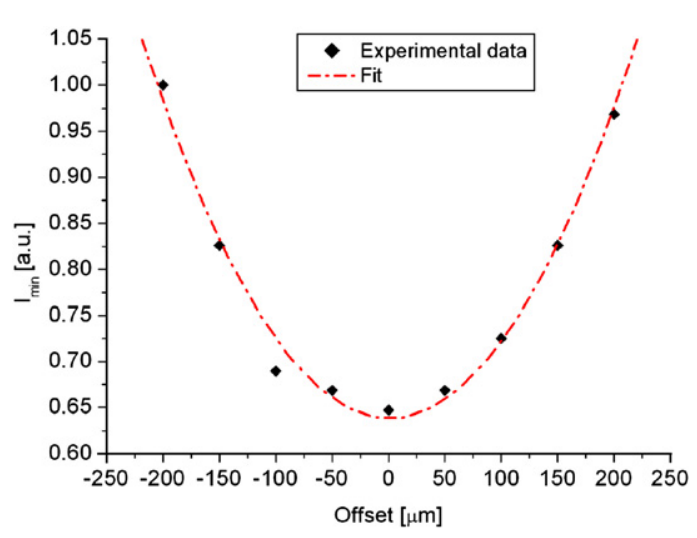

Fig. 12. Measured dependencies of ODR minimum intensity as function of the displacement of the beam within the slit.

dure. For this measurement, the projection of the ODR angular distribution image is shown in Fig. 13 (black squares). A simulation which takes into account a Gaussian distributed beam with $\sigma_{y}=80 \mu \mathrm{m}$, compatible with the one given from fitting the beam image profile and $\sigma_{y}^{\prime}=125 \mu \mathrm{rad}$, shows a very good qualitative agreement with the measured ODR projection (Fig. 13, line).

From this measurement and in particular from the values of beam size and angular divergence given by the sim-

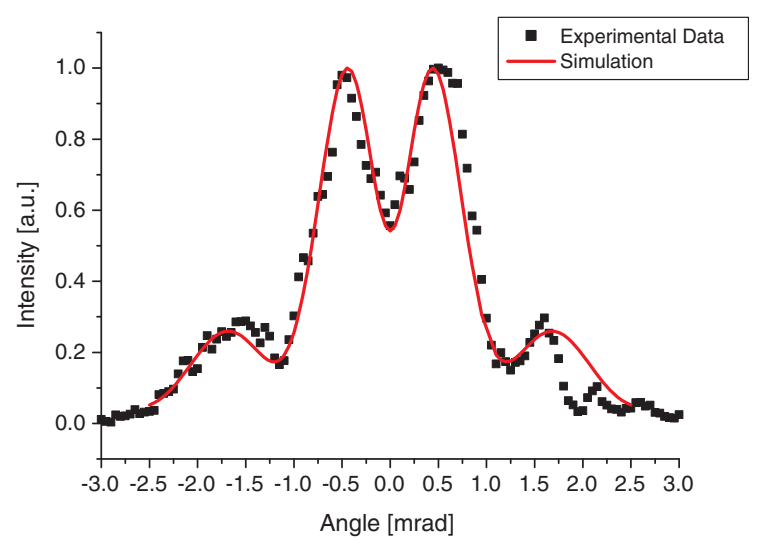

Fig. 13. ODR angular distribution: 25 bunches per macropulse, $0.7 \mathrm{nC}$ per bunch, $2 \mathrm{~s}$ exposure time, $5 \mathrm{~Hz}$ repetition rate, $0.5 \mathrm{~mm}$ slit. Polarizer and $800 \mathrm{~nm}$ filter are inserted.

ulation, the normalized vertical transverse emittance can be extracted. Assuming that the beam is in a waist, it is estimated to be about $12 \mathrm{~mm}$ mrad. The disagreement with the typical value of the emittance for FLASH $(\approx 2 \mathrm{~mm} \mathrm{mrad}$ ) can be ascribed to the fact that we are in the by-pass line where the beam conditions are very far from the nominal ones and, in particular, optimized for our experiment. 


\subsection{Discussion}

The main limitations during both periods of measurements were given by the background and the large amount of hot spots which did not allow us to increase the exposure time of the camera.

The background image is every time acquired by moving the beam out of the screen by means of steering magnets upstream of the target. However, since the steered beam hits the vacuum pipe, this procedure gives rise to a large amount of X-rays. In order to increase the signal-to-noise ratio, a large number of images has been recorded. The images have been then off-line processed with a custom LabView tool in order to first eliminate X-rays and then subtract the background from the signal. This tool becomes mandatory for the analysis of ODR signals which, being of the same order of magnitude and even weaker than the background, are covered by it.

\section{Summary and conclusions}

The presence of an unavoidable background, together with non-optimal beam conditions, was a severe limitation for a rigorous reconstruction of the beam parameters from the ODR angular distribution.

Nevertheless, we were able, even at this low energy, to detect a clear signature of optical diffraction radiation which has allowed us to prove a satisfactory agreement with simulations. In addition, the effort put in the data post-processing to clean images from X-rays and to properly subtract the synchrotron radiation background has permitted to extract a preliminary beam emittance from the collected data.

Since FLASH is operated most of the time as a FEL user facility, the beam time for dedicated studies, like our experiment, is limited.

Our next and last measurements are planned in January 2008 with an electron beam energy of about $1 \mathrm{GeV}$, which means more than a factor of 2 in the radiation intensity. Furthermore, to reduce the influence of the radiation background we mounted a new thin shield in front of the target and replaced the holder with one which is machined such that reflections are strongly suppressed. The higher energy and the improved layout, together with better conditions of the beam, give us confidence in completing the experiment successfully.

\section{Acknowledgements}

We thank DESY and FLASH team for the opportunity to perform our experiment. Special thanks are to FLASH shift crews for tuning the linac for beam conditions required for the experiment. We thank also R. Sorchetti and L. Cacciotti (INFN-LNF) for their fundamental technical assistance and B. Polzin (DESY) for his support on control system issues.

\section{References}

[1] $<$ www.linearcollider.org $>$.

[2] F. Richard et al. (Ed.), TESLA Technical design report, March 2001, DESY-2001-011.

[3] LCLS Conceptual design report <www-ssrl.slac.stanford.edu/lcls/ cdr>.

[4] SPARC Group (LNF-INFN, Roma1-INFN, Roma2-INFN, ENEA, CNR, Milano-INFN), Technical design report, January 2004 $<$ www.lnf.infn.it/acceleratori/sparc/>.

[5] P. Karataev, S. Araki, R. Hamatsu, H. Hayano, T. Muto, G. Naumenko, A. Potylitsyn, N. Terunuma, J. Urakawa, Phys. Rev. Lett. 93 (2004) 244802.

[6] A.H. Lumpkin, W.J. Berg, N.S. Sereno, D.W. Rule, C.-Y. Yao, Phys. Rev. ST Accel. Beams 10 (2007) 022802.

[7] M. Castellano, A new non-intercepting beam size diagnostics using diffraction radiation from a slit, Nucl. Instr. and Meth. Phys. Res. A394 (1997) 275.

[8] M. Castellano, Diffraction Radiation as a Non-Intercepting Diagnostics for TTF: the Bunch Length Measurement, TESLA 96-08.

[9] M.L. Ter-Mikaelian, High-Energy Electromagnetic Processes in Condensed Media, Wiley-Interscience, New York, 1972.

[10] E. Chiadroni, PhD Thesis, TESLA FEL 2006-9 $(<$ http://flash.desy.de/reports_publications/tesla_fel_reports/tesla_fel_2006/ index_eng.html $>$ ).

[11] M. Born, E. Wolf, Principles of Optics, Pergamon Press, 1965.

[12] E. Cianci et al., Micromachined silicon slit for beam diagnostic in particle accelerator, Proc. SPIE 4557 (2001) 242.

[13] D. Xiang, W.-H. Huang, Nucl. Instr. and Meth. B 254 (2007) 165. 